\title{
Tests giving an aetiological diagnosis in pulmonary disease in patients infected with the human immunodeficiency virus
}

\author{
R F Miller, T R Leigh, J V Collins, D M Mitchell
}

The use of non-invasive tests in the evaluation of pulmonary disease in patients with AIDS is reviewed in the accompanying article in this series $\left(\mathrm{p} 57^{1}\right)$. Although non-invasive tests are important, they will rarely yield a specific diagnosis. Techniques that enable an aetiological diagnosis to be made are discussed in this article-namely, sputum induction, fibreoptic bronchoscopy with bronchoalveolar lavage or transbronchial lung biopsy, and open lung biopsy.

Before discussing these we might ask if it is important to establish an aetiological diagnosis in patients with AIDS with respiratory symptoms as nearly all will have pneumocystis pneumonia. In centres where the technique of induced sputum examination has been developed this is hardly an issue as the procedure for inducing sputum is simple and non-invasive. In centres relying on bronchoscopy to establish an aetiological diagnosis, it has been argued that most patients may be treated empirically without recourse to bronchoscopy. The advantage of this approach is that a potentially hazardous procedure for the patient is avoided and demands on the bronchoscopy and laboratory services are reduced. For some patients bronchoscopy is probably unnecessary. Some will be too ill for the procedure at presentation and others may have had previous episodes of pneumocystis pneumonia that clearly resemble the current episode. There is also probably little point in confirming the diagnosis by bronchoscopy in patients for whom bronchoscopy could not be arranged at presentation and who are improving with treatment. On the other hand, for many patients an accurate diagnosis is desirable. An early aetiological diagnosis allows early intervention with specific treatment and this will produce a better outcome. If the patient deteriorates despite treatment and the diagnosis is certain, alternative treatment can be instituted with confidence. Some patients may have more than one pathogen (for example Pneumocystis carinii and Mycobacterium tuberculosis) and this will be missed if bronchoscopy is omitted. In cases where a respiratory illness such as pneumocystis pneumonia may be an AIDS defining condition, certainty is required because of the important implications for the patient and the subsequent treatment (that is, prophylaxis against further episodes of pneumocystis pneumonia and zidovudine treatment). With increasing use of prophylactic treatment pneumocystis pneumonia may become less common or its clinical manifestations may be modulated, making a firm diagnosis more important. Clearly several factors need to be considered in an individual patient, and each case should be judged on its merits.

\section{Induced sputum}

Patients with AIDS and pulmonary disease do not usually produce sputum spontaneously, so that lung washings or tissue need to be obtained to reach a diagnosis. Until recently this usually required an invasive procedure such as fibreoptic bronchoscopy. Before the advent of AIDS pneumocystis pneumonia was diagnosed very occasionally by sputum examination. For example, in a review of 194 episodes of pneumocystis pneumonia in patients without AIDS in 1974, the diagnostic yield for sputum was only $6 \% .^{2}$ In an attempt to avoid invasive procedures such as fibreoptic bronchoscopy, several centres in the United States have developed a technique to generate sputum samples adequate for diagnosis. ${ }^{3}$

To induce sputum production the patient inhales $20-30 \mathrm{ml}$ of hypertonic saline $(3 \%-5 \%)$ in the form of a fine mist generated by a nebuliser over 10-20 minutes. Small droplets are deposited at relatively peripheral sites in the airways, whereas larger droplets are deposited more centrally. The hypertonic solution irritates the airways and induces bronchial secretions. The hypertonicity of the saline also tends to draw water into the airways from the interstitial fluid by osmosis. Casts from alveoli filled with inflammatory exudate and pneumocystis organisms are mobilised by the ingress of fluid and move centrally. Repeated deep coughing by the patient mobilises the material into the trachea and through the mouth. It is important that sputum is free from contamination with food debris and squamous cells from the upper respiratory tract, as this material can take up the Giemsa stain and may make analysis difficult. Patients should therefore be starved from the night before the procedure and should brush their teeth carefully with water and then rinse their mouth 
thoroughly immediately before the procedure. An ultrasonic nebuliser (for example, Devilbiss Ultraneb 99) with a high flow rate (up to $3 \mathrm{ml} /$ $\mathrm{min}$ ) is more efficient in inducing sputum than an ordinary jet nebuliser. A face mask is preferable to a mouthpiece because the larger saline droplets are deposited in the nose and do not irritate the trachea and larynx. The material coughed up after about 10 minutes of inhalation is mainly from the central airways and contains residual debris and usually fewer cysts than the sputum obtained later. This first sample is best discarded. Sputum obtained after a further 10 minutes of inhalation is sent for analysis and is most likely to contain material from distal airways. Purulent sputum samples are unlikely to yield $P$ carinii and suggest a bacterial cause for the pneumonia. $P$ carinii is usually found in clear watery specimens resembling saliva. Inexperienced laboratories may mistakenly discard such specimens as being unsuitable for analysis. Most patients tolerate the induced sputum procedure, though some find inhaling saline mist unpleasant and others develop nausea. ${ }^{45}$

In an early study ${ }^{6}$ induced sputum was obtained before bronchoscopy and transbronchial biopsy in 43 patients with AIDS or at high risk for AIDS who presented with respiratory symptoms. The induced sputum samples were cytocentrifuged and material from the pellet was smeared on the slides and stained with silver methionine to outline the cyst walls of $P$ carinii. Of the 20 patients shown to have pneumocystis pneumonia after bronchoscopy, 11 had a positive result from induced sputum. Furthermore, bronchoscopy did not identify any additional pathogens in those with $P$ carinii in the induced sputum. In another study $^{7}$ induced sputum was obtained from 32 patients at high risk for AIDS who had respiratory symptoms and either an abnormal chest radiograph, reduced transfer factor for carbon monoxide, or an abnormal gallium scan. Sputum samples were not cytocentrifuged but were smeared directly and processed with Giemsa to stain trophozoites and precyst forms of $P$ carinii. These were found in 14 of the 32 patients, and in none of these was any other organism identified. The 18 patients with a negative result from induced sputum underwent bronchoscopy with bronchoalveolar lavage and transbronchial biopsy. $P$ carinii was found in 11 . In the remaining seven patients other diagnoses, including Kaposi's sarcoma, were made. The sensitivity of induced sputum in identifying $P$ carinii was $14 / 25(56 \%)$, very similar to that found by Pitchenik et al. ${ }^{6}$ Despite these encouraging results, other centres have not been able to repeat these findings, mainly for technical reasons. Although relatively simple, the techniques require meticulous attention to detail if they are to succeed. ${ }^{5}$ Care in obtaining the sputum sample has already been mentioned. It is also important that samples reach the laboratory quickly for good results, and skilled and patient laboratory staff are required to process and examine the specimens. Scanning of samples for $P$ carinii requires particular expertise and diligence; most induced sputum samples contain 100 times fewer organisms than the corresponding lavage fluid samples. Liquefaction of the sputum samples by means of dithiothreitol with subsequent centrifugation and concentration of the alveolar casts containing pneumocystis increases the diagnostic yield further. ${ }^{8}$ Other pathogens may be detected by standard staining procedures (for example, Giemsa staining), such as Candida albicans, Cryptococcus neoformans, Histoplasma capsulatum, and cytomegalovirus inclusion bodies, and mycobacteria by Ziehl-Nielsen staining. Both induced sputum and lavage fluid should be sent routinely for culture.

Indirect immunofluorescent staining of induced sputum samples using monoclonal antibodies to pneumocystis has been shown to give a higher diagnostic yield than conventional staining. A recent study ${ }^{9}$ showed a diagnostic yield of $80 \%$ with routine staining (toluidine blue $\mathrm{O}$ ) and $92 \%$ with immunofluorescence. The development of pneumocystis specific DNA probes may further increase the sensitivity of this technique. ${ }^{1011}$ Induced sputum analysis is a useful diagnostic technique and in centres where it has been developed will greatly reduce the need for fibreoptic bronchoscopy. Fibreoptic bronchoscopy will continue to be necessary for patients with a negative result from induced sputum and for patients whose clinical diagnosis and response to treatment are at variance with the cytological or microbiological diagnosis obtained from induced sputum. Finally, considerable time, effort, and financial outlay is required to develop the technique of induced sputum, and this can only be justified in centres seeing a large number of patients with AIDS. In centres with few such patients bronchoscopy will remain the investigation of choice.

\section{Bronchoscopy}

Fibreoptic bronchoscopy is well established as a diagnostic tool for a large range of pulmonary disorders ${ }^{1213}$ and the techniques of bronchoalveolar lavage $e^{14} 15$ and transbronchial biopsy $^{16} 17$ are widely used. Lavage was shown to be an effective method of diagnosing pneumocystis pneumonia before the AIDS epidemic. ${ }^{1819}$ Bronchoalveolar lavage combined with transbronchial lung biopsy provides a high diagnostic yield for all the opportunist infections that affect the lung in patients with AIDS. A large retrospective series early in the AIDS epidemic reported that $91 \%$ of all pulmonary infections could be diagnosed by a combination of bronchoalveolar lavage and transbronchial lung biopsy..$^{20}$ In a further study the diagnostic yields were $88 \%$ for transbronchial lung biopsy and $85 \%$ for bronchoalveolar lavage, with a combined overall yield of $94 \% .21$ Other studies have supported these findings. $22-25$ Diagnostic yields are particularly good with both techniques for $P$ carinii. Many groups now consider that the results from lavage alone are sufficiently good to justify the omission of routine transbronchial lung biopsy $y^{26-28}-$ not surprisingly, as bronchoalveolar lavage 
presumably samples many alveoli, far more than are represented in a small transbronchial biopsy specimen. Bronchoalveolar lavage alone will not, however, identify lymphocytic interstitial pneumonitis ${ }^{29}{ }^{30}$ or non-specific interstitial pneumonitis ${ }^{31}$ and these diagnoses will be missed unless transbronchial or open lung biopsy is performed.

Omission of transbronchial lung biopsy simplifies and shortens the bronchoscopic procedure and also eliminates the complications associated with transbronchial lung biopsy. When transbronchial lung biopsy is carried out via a fibreoptic bronchoscope in patients not suffering from AIDS it is safe with a low incidence of complications ${ }^{1732}$; but patients with AIDS undergoing transbronchial lung biopsy have an increased rate of complications-the risk appearing particularly high in patients with pneumocystis pneumonia, ${ }^{33}$ whether or not fluoroscopic screening is used. ${ }^{34}$ The reported incidence of pneumothorax has ranged from $4 \%$ to $20 \%{ }^{24}{ }^{33} 34$ Haemorrhage is also more common and may be fatal. ${ }^{28}$

During bronchoscopy the endobronchial lesions of Kaposi's sarcoma may be seen. ${ }^{3536}$ These are either flat, resembling the submucosal haemorrhages induced by traumatic bronchoscopy, or raised, perhaps even producing partial occlusion of bronchi. Bronchial lesions of Kaposi's sarcoma are usually seen in patients with extensive lesions at other sites, particularly the skin, thus making diagnostic biopsy unnecessary. Kaposi's sarcoma is a vascular tumour, so successful biopsy of an endobronchial lesion may produce substantial haemorrhage.

The timing of fibreoptic bronchoscopy in relation to starting antimicrobial treatment is probably not critical as the cyst forms of pneumocystis persist for days or even weeks after treatment is started. ${ }^{378}$ Treatment may therefore be started before bronchoscopy on the basis of clinical suspicion, pending a firm diagnosis.

The technique of bronchoscopy in patients with AIDS is essentially the same as in patients without AIDS (control of infection measures are discussed in the first article in this series, (October 1989;44:778-83). Patients with AIDS often cough excessively and have irritable airways. The routine use of nebulised lignocaine ( $6 \mathrm{ml}$ of a $4 \%$ solution) with the premedication has been advocated ${ }^{39}$ and a short acting benzodiazepine such as midazolam is often required ${ }^{40}$ during the procedure. There may be a considerable fall in arterial oxygen saturation during bronchalveolar lavage. ${ }^{41}$ Patients with AIDS and pneumocystis pneumonia may have a low initial oxygen saturation, which may fall further during bronchoalveolar lavage. The use of a transcutaneous oxygen saturation meter (oximeter) during the procedure helps to determine supplemental oxygen requirements. Additional oxygen may be delivered by nasal cannulae ${ }^{42}$ or by a tight fitting oxygen mask delivering $60 \%$ oxygen, the bronchoscope being passed through a small hole cut in the side of the mask.

After the airways have been inspected bron- choalveolar lavage is carried out most conveniently from the right middle lobe with warmed saline aliquots of 3-60 ml to a total volume of $120-180 \mathrm{ml}^{192740}$ If transbronchial biopsy is performed specimens are normally taken from the right lower lobe. ${ }^{22}$ Laboratory processing of the bronchoalveolar lavage fluid should include microbiological culture, cytological examination, and staining specifically for pneumocystis. Transbronchial biopsy specimens should be placed in sterile saline for culture and in formol saline for histopathology.

\section{Open lung biopsy}

Open lung biopsy provides a high diagnostic yield in patients with diffuse pulmonary disease. ${ }^{43}$ The specimen obtained by wedge resection is large enough to provide plenty of material for histological examination and culture, and the procedure has a low morbidity, ${ }^{44}$ though some patients who are hypoxaemic before the procedure will require assisted ventilation afterwards. Before the AIDS epidemic and the ready availability of fibreoptic bronchoscopy open lung biopsy was used successfully to diagnose pneumocystis pneumonia and other pulmonary infections in immunosuppressed patients. ${ }^{456}$ It was also used successfully to diagnose opportunist lung infections at the beginning of the AIDS epidemic in 1981 and 1982. It is now rarely necessary for patients with AIDS because of the high success rate of bronchoalveolar lavage and transbronchial lung biopsy. Open lung biopsy may be required if bronchoscopy fails to provide a diagnosis, ${ }^{47}$ or when fibreoptic bronchoscopy is contraindicated and may be expected to give a high diagnostic yield. ${ }^{48}$

\section{Fine needle aspiration biopsy}

Percutaneous needle aspiration provides a rapid and accurate method of cytological and microbiological diagnosis of pulmonary infection in children and adults. ${ }^{49}{ }^{50}$ In patients with AIDS the procedure is associated with a high incidence of complications, including pneumothorax and cardiac arrest, and cannot be recommended. ${ }^{51}$

\section{Conclusions}

Invasive tests enable a specific aetiological diagnosis to be made in virtually all HIV positive patients who present with respiratory symptoms. Sputum induction may provide an aetiological diagnosis without the need for more invasive tests and should be done first if possible. Meticulous care and additional resources are needed for this technique to be applied successfully. The use of mucolytic agents and immunofluorescent staining with monoclonal antibodies increases the diagnostic yield for $P$ carinii from induced sputum and the use of DNA probes for pneumocystis in the near future should greatly enhance the sensitivity and specificity of the technique. If the technique of sputum induction is not available or the result is negative fibreoptic broncho- 
scopy will usually be considered necessary. At bronchoscopy bronchoalveolar lavage is carried out routinely; the addition of transbronchial biopsy adds little to the diagnostic yield and as it is associated with a high incidence of complications is now carried out rarely. If, despite bronchoscopy, no specific diagnosis is made and a firm diagnosis is clinically imperative it may be necessary to resort to open lung biopsy.

1 Millar AB, Mitchell DM. Non-invasive investigation of pulmonary disease in patients positive for the human pulmonary disease in patients positive for the

2 Walzer PD, Perl DP, Krogstad DJ, Rawson PG, Schultz MG. Pneumocystis carinii pneumonia in the United States: MG. Pneumocystis carinii pneumonia in the United States: Med 1974;80:83-93.

3 Luce JM. Sputum induction in the acquired immunodeficiency syndrome. Am Rev Respir Dis 1986;133:513-4.

4 Leigh TR, Parsons P, Hume C, Husain OAN, Gazzard BG, Collins JV. Induced sputum: a sensitive test for diagnosing Pneumocystis carinii pneumonia in AIDS patients. Lancet 1989;ii:205-6.

5 Leigh TR, Parsons P, Hume C, Husain OAN, Collins JV. Sputum induction as a reliable method of diagnosing Pneumocystis carinii pneumonia in AIDS patients [absPneumocystis carinii pneum
tract]. Thorax 1989;44:318.

6 Pitchenik AE, Gangei R, Torres A, Evans DA, Rubin E, Baier H. Sputum examination for the diagnosis of Pneumocystis carinii pneumonia in the acquired immunodeficiency syndrome. Am Rev Respir Dis 1986;133:226-9.

7 Bigby T, Margolskee D, Curtis J, et al. The usefulness of induced sputum in the diagnosis of Pneumocystis carinii pneumonia in patients with the acquired immunodeficiency syndrome. Am Rev Respir Dis 1986;133:515-8.

8 Zaman MK, Wooken OJ, Supramanya B, et al. Rapid noninvasive diagnosis of Pneumocystis carinii from ind

9 Kovacs JA, Ng VL, Masur H, Leoung G, et al. Diagnosis of Pneumocystis carinii pneumonia: improved detection in Pneumocystis carinii pneumonia: improved detection in
sputum with use of monoclonal antibodies. $N$ Engl J Med sputum with use of

10 Wakefield AE, Hopkin JM, Burns J, Hipkiss JB, Stewart TJ, Moxon ER. Cloning of DNA from Pneumocystis carinii. Infect Dis 1988;158:859-62.

11 Tanabe K, Fuchimoto M, Egawa-K, Nakamura Y. Use of Pneumocystis carinii genomic DNA clones for DNA hybridization analysis of infected human lungs. $J$ Infect Dis 1988;3:593-6.

12 Mathey RA, Moritz ED. Invasive procedures for diagnosing pulmonary infection. Clin Chest Med 1987;2:3-18.

13 Fulkerson WJ. Fibreoptic bronchoscopy. N Engl J Med 1984;311:511-5.

14 Hunninghake GW, Godek JE, Karvanani O, et al. Inflammatory and immune processes in the human lung in health and disease evaluation by bronchoalveolar lavage. $\mathrm{Am} \mathrm{J}$ Pathol 1979;97:149-206.

15 Hopkin JM, Turney JH, Young JA, et al. Rapid diagnosis of obscure pneumonia in immunosuppressed renal patients by cytology of alveolar lavage fluid. Lancet 1983;ii 299-301.

16 Springmeyer SC, Silvestri RC, Sale GE, et al. The role of transbronchial biopsy for the diagnosis of diffuse pneumonias in immuno-compromised transplant pneumonias in immuno-compromised

17 Mitchell DM, Emerson CJ, Collins JV, Stableforth DE. Transbronchial lung biopsy with the fibreoptic broncho-
scope: Analysis of results in 433 patients. Br J Dis Chest scope: Analysis of

18 Drew WL, Finley JN, Mintz L, Klein HZ. Diagnosis of Pneumocystis carinii pneumonia by bronchopulmonary lavage. JAMA 1974;230:713-5.

19 Kelley J, Landis JN, Davis GS, et al. Diagnosis of pneumonia due to Pneumocystis by subsegmental pulmonary lavage via the fibreoptic bronchoscope. Chest 1978 74:24-8.

20 Murray JF, Felton CP, Garay S, et al. Pulmonary complications of the acquired immunodeficiency syndrome. Report of the National Heart Lung and Blood Institute Workshop. N Engl J Med 1984;310:1682-8.

21 Stover DE, White DA, Romano PA, et al. Diagnosis of pulmonary disease in the acquired immune deficiency syndrome: roles of bronchoscopy and bronchoalveolar syndrome: roles of bronchoscopy and bro

22 Broddus C, Dake MD, Stulbarg MA, et al. Bronchoalveolar lavage and transbronchial biopsy for the diagnosis of pulmonary infections in the acquired immunodeficiency syndrome. Ann Intern Med 1985;102:747-52.

23 Rosen MJ, Tow TW, Teirstein AS, et al. Diagnosis of pulmonary complications of the acquired immune deficiency syndrome. Thorax 1985;40:571-5.

24 Orenstein M, Webber CA, Cash M, et al. Value of bron- choalveolar lavage in the diagnosis of pulmonary infection in the acquired immune deficiency syndrome. Thorax 1986;41:345-9.

25 Gal AA, Klatt EC, Koss MN, Strigle SM, Boyle CT. The effectiveness of bronchoscopy in the diagnosis of Pneumocystis carinii and cytomegalovirus pulmonary infections in acquired immunodeficiency syndrome. Arch infections in acquired immunodeficie
Pathol Lab Med 1987;111:238-41.

26 Ognibene FP, Shelhamer J, Gill V, et al. The diagnosis of Pneumocystis carinii pneumonia in patients with acquired mmunodeficiency syndrome using subsegmental bronchoalveolar lavage. Am Rev Respir Dis 1984;129:929-32.

27 Golden JA, Hollander H, Stubarg MS, Gamsu G. Bronchoalveolar lavage as the exclusive diagnostic modality for Pneumocystis carinii pneumonia. Chest 1986;90:18-22.

28 Griffiths MM, Kocjan G, Miller RF, Godfrey-Faussett P. Diagnosis of pulmonary disease in human immunodeficiency virus infection: role of transbronchial biopsy and bronchoalveolar lavage. Thorax 1989;44:554-8.

29 Morris JC, Rosen MJ, Marcheosky A, Teirstein AS. Lymphocytic interstitial pneumonia in patients at risk for the acquired immune deficiency syndrome. Chest 1987;91: 63-7.

30 Oldham SAA, Castino M, Jacobson FL, et al. HIV associated lymphocytic interstitial pneumonia. Radiologic manifestations and pathologic correlation. Radiology 1989;170:83-7.

31 Ognibene FP, Masur H, Rogers P, et al. Non specific interstitial pneumonitis without evidence of Pneumocystis carinii in asymptomatic patients infected with human immunodeficiency virus (HIV). Ann Intern Med 1988; 109:874-9.

32 Simpson FG, Arnold AG, Purvis A, et al. Postal survey of bronchoscopic practice by physicians in the United Kingdom. Thorax 1986;41:311-7.

33 Miller RF, Millar AB, Semple SJG. Complications of fibreoptic bronchoscopy in HIV-1 antibody positive patients undergoing investigations for pulmonary disease [abstract]. Thorax 1988;43:847P.

34 Milligan SA, Luce JM, Golden J, et al. Transbronchia biopsy without fluroscopy in patients with diffuse roentgenographic infiltrates and the acquired immunogenographic infiltrates and the acquired immuno-

35 Meduri GU, Stover DE, Lee M, et al. Pulmonary Kaposi's sarcoma in the acquired immunodeficiency syndrome. $\mathrm{Am}$ J Med 1986;81:11-8.

36 Ognibene FP, Steis RG, Macher AM, et al. Kaposi's sarcoma causing pulmonary infiltrates and respiratory failure in the acquired immunodeficiency syndrome. $A n n$ Intern Med 1985;102:471-5

37 DeLorenzo LJ, Maguire GP, Wormser GP, Davidian MM, Stone DJ. Persistence of Pneumocystis carinii pneumonia in the acquired immunodeficiency syndrome. Evaluation of therapy by follow-up transbronchial lung biopsy. Chest 1985;88:79-83.

38 Shelhamer JH, Ognibene FP, Macher AM, et al. Persistence of Pneumocystis carinii in lung tissue of acquired immunodeficiency syndrome patients treated for immunodeficiency syndrome patients treated for
Pneumocystis pneumonia. Am Rev Respir Dis 1984; Pneumocystis
130:1161-5.

39 Mitchell DM. Diagnostic problems in AIDS and the lung. Respir Med 1989;83:9-14

40 Miller RF, Millar AB, Weller IVD, Semple SJG. Empirical treatment without bronchoscopy for Pneumocystis carinit pneumonia in AIDS. Thorax 1989;44:559-64.

41 Hendy MS, Bateman JR, Stableforth DE. The influence of transbronchial lung biopsy and bronchoalveolar lavage on arterial blood gas changes occurring in patients with diffuse interstitial lung disease. $B r J$ Dis Chest 1984;78: 363-8.

42 Hussain SA, Meyer CA, Saul R. Delivery of oxygen during fibreoptic bronchoscopy under local anaesthesia. Int Surg 1977;62:495-8.

43 Sattersfield JR, McLaughlin JS. Open lung biopsy in diagnosing pulmonary infiltrates in immunosuppressed patients. Ann Thorac Surg 1979;28:359-62.

44 Venn GE, Kay PH, Midwood CJ, Goldstraw P. Open lung biopsy in patients with diffuse pulmonary shadowing. Thorax 1985;40:931-5.

45 Michaelis LL, Leight GS, Powell RD, DeVita VT Pneumocystis pneumonia: the importance of early open lung biopsy. Ann Surg 1976;183:301-6.

46 Rosen PP, Martini N, Armstrong D. Pneumocystis carini pneumonia: diagnosis by lung biopsy. Am J Med 1975;58: pneumon

47 Millar AB. Respiratory manifestations of AIDS. Br J Hosp Med 1988;3:204-15.

48 Fitzgerald W, Bevelaqua FA, Garay SM, Aranda CP. The role of open lung biopsy in patients with the acquired immunodeficiency syndrome. Chest 1987;91:659-61.

49 Mimica I, Omoso E, Howard JE, Ledermann GW. Lung puncture in the aetiological diagnosis of pneumonia. A study of 543 infants and children. Am J Dis Child 1971;
122:278-82. Palmer DL, Davidson M, Lusk R. Needle aspiration of the lung in complex pneumonias. Chest 1980;78:16-21.

51 Craddock C, Pastvol G, Bull R, et al. Cardiorespiratory arrest and autonomic neuropathy in AIDS. Lancet 1987; ii: $16-8$. 